\title{
Multiple nitrogen reservoirs in a protoplanetary disk at the epoch of comet and giant planet formation
}

\author{
P. Hily-Blant ${ }^{1}$, V. Magalhaes de Souza ${ }^{2}$, J. Kastner ${ }^{3}$, and T. Forveille ${ }^{1}$ \\ 1 Univ. Grenoble Alpes, CNRS, IPAG, 38000 Grenoble, France \\ e-mail: pierre.hily-blant@univ-grenoble-alpes.fr \\ 2 IRAM, 300 Rue de la Piscine, Grenoble, France \\ 3 Chester F. Carlson Center for Imaging Science, School of Physics \& Astronomy, and Laboratory for Multiwavelength Astrophysics, \\ Rochester Institute of Technology, 54 Lomb Memorial Drive, Rochester, NY 14623, USA
}

Received 20 September 2019 / Accepted 4 November 2019

\begin{abstract}
The isotopic ratio of nitrogen measured in primitive Solar System bodies shows a broad range of values, the origin of which remains unknown. One key question is whether these isotopic reservoirs of nitrogen predate the comet formation stage or are posterior to it. Another central question is elucidating the processes that can produce the observed variations in the ${ }^{14} \mathrm{~N} /{ }^{15} \mathrm{~N}$ isotopic ratio. Disks that orbit pre-main-sequence ( T Tauri) stars provide unique opportunities for observing the chemical content of analogs of the protosolar nebula and therefore for building a comprehensive scenario that can explain the origin of nitrogen in the Solar System and in planetforming disks. With ALMA, it has become possible to measure isotopic ratios of nitrogen-bearing species in such environments. We present spectrally and spatially resolved observations of the hyperfine structure of the 4-3 rotational transition of $\mathrm{HCN}$ and its main isotopologs $\mathrm{H}^{13} \mathrm{CN}$ and $\mathrm{HC}^{15} \mathrm{~N}$ in the disk orbiting the $8 \mathrm{Myr}$ old T Tauri star TW Hya. The sensitivity allows directly measuring the $\mathrm{HCN} / \mathrm{H}^{13} \mathrm{CN}$ and $\mathrm{HCN} / \mathrm{HC}^{15} \mathrm{~N}$ abundance ratios with minimal assumptions. Averaged spatially over the disks, the ratios are $86 \pm 4$ and $223 \pm 21$, respectively. The latter value is significantly lower than the $\mathrm{CN} / \mathrm{C}^{15} \mathrm{~N}$ ratio of $323 \pm 30$ in this disk and thus provides the first evidence that two isotopic reservoirs of nitrogen are present in a disk at the stage of giant planet and comet formation. Furthermore, we find clear evidence for an increase in the ratio of $\mathrm{HCN}$ to $\mathrm{HC}^{15} \mathrm{~N}$ with radius. The ratio in the outer disk, at 45 au, is $339 \pm 28$, in excellent agreement with direct measurements in the local interstellar medium, and with the bulk nitrogen isotopic ratio predicted from galactic evolution calculations. In the comet formation region at $r=20$ au, the ratio is a factor $\approx 3$ lower, $121 \pm 11$. This radial increase qualitatively agrees with the scenario in which selective photodissociation of $\mathrm{N}_{2}$ is the dominant fractionation process. However, our isotopic ratios and kinetic temperature of the $\mathrm{HCN}$-emitting layers quantitatively disagree with models of nitrogen chemistry in disks.
\end{abstract}

Key words. comets: general - protoplanetary disks

\section{Introduction}

The isotopic ratio of nitrogen, ${ }^{14} \mathrm{~N} /{ }^{15} \mathrm{~N}$, in various bodies of the Solar System (meteorites, comets, planets, etc.) shows the largest variations among the most abundant constituents (carbon and oxygen), with values ranging from $\sim 50$ in submicron grains that are immersed in a chondrite matrix (so-called hotspots) to 441 in the Sun and Jupiter (Bonal et al. 2010; Marty et al. 2011; Fouchet et al. 2000; Hily-Blant et al. 2013). When, where, and how these isotopic reservoirs of nitrogen formed during the evolution from a molecular cloud to planetary systems remains to be determined. Comets provide a special case among primitive cosmomaterials: regardless of the cometary type (short or long period) and of the carrier of nitrogen that is observed in their coma $\left(\mathrm{CN}, \mathrm{HCN}, \mathrm{NH}_{2}, \mathrm{NH}_{3}, \mathrm{~N}_{2}\right.$, or $\left.\mathrm{NO}\right)$, the isotopic ratio is consistently found to be $\approx 140$ (Wampfler et al. 2018; Hily-Blant et al. 2017, hereafter Paper I, and references therein). This is lower by a factor three than the ratio of the bulk proto-Sun, which is 441. This well-established observational fact remains unexplained.

Evolved protoplanetary disks at the stage of giant planet and comet formation provide unique opportunities for investigating how and when this ${ }^{15} \mathrm{~N}$-rich reservoir of nitrogen was built up. The nitrogen isotopic ratio was measured via rare isotopologs ${ }^{1}$ of $\mathrm{HCN}$, specifically, $\mathrm{H}^{13} \mathrm{CN}$ and $\mathrm{HC}^{15} \mathrm{~N}$, for a small sample of disks with the ALMA interferometer (Guzmán et al. 2017), yielding an $\mathrm{HCN} / \mathrm{HC}^{15} \mathrm{~N}$ between $83 \pm 32$ and $156 \pm 71$, and a mean value of $111 \pm 19$, under the assumption that $\mathrm{HCN} / \mathrm{H}^{13} \mathrm{CN}=70$. On the other hand, a direct measurement, one that does not involve assuming a ${ }^{12} \mathrm{C} /{ }^{13} \mathrm{C}$ ratio, of $\mathrm{CN} / \mathrm{C}^{15} \mathrm{~N}$ in the TW Hya system yielded a significantly higher ratio, $323 \pm 30$ (Paper I). Based on this value and on directly measured values in the dense local interstellar medium (ISM), a value of ${ }^{14} \mathrm{~N} /{ }^{15} \mathrm{~N}=330 \pm 30$ was proposed for the present-day bulk solar neighborhood. This also agrees excellently well with predictions from the galactic chemical evolution model of Romano et al. (2017) for the local ISM. A new picture thus emerged in which $\mathrm{HCN}$ and $\mathrm{CN}$ trace two isotopic reservoirs of nitrogen that are present in analogs of the protosolar nebula (PSN) at the time of comet formation, with $\mathrm{HCN}$ representing a ${ }^{15} \mathrm{~N}$-enriched reservoir of nitrogen similar to that recorded in Solar System comets.

\footnotetext{
${ }^{1}$ Hereafter, the three isotopologs $\mathrm{H}^{12} \mathrm{C}^{14} \mathrm{~N}, \mathrm{H}^{13} \mathrm{C}^{14} \mathrm{~N}$, and $\mathrm{H}^{12} \mathrm{C}^{15} \mathrm{~N}$, are denoted $\mathrm{HCN}, \mathrm{H}^{13} \mathrm{CN}$, and $\mathrm{HC}^{15} \mathrm{~N}$, respectively.
} 

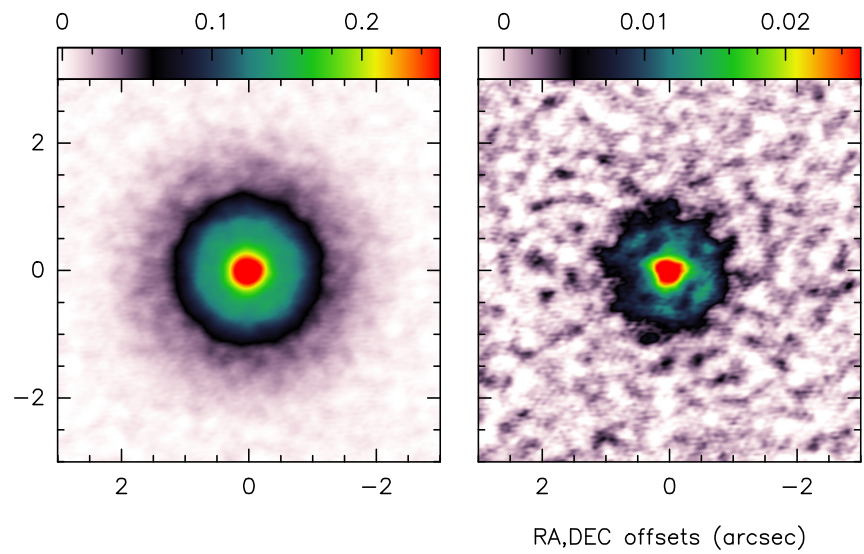

However, $\mathrm{HCN} / \mathrm{HC}^{15} \mathrm{~N}$ was only measured indirectly, and moreover, this was not done in TW Hya, therefore clear-cut evidence for this scenario was lacking.

To elucidate how the various nitrogen reservoirs in the Solar System were formed requires identifying the processes that lead to variations of ${ }^{14} \mathrm{~N} /{ }^{15} \mathrm{~N}$ (Hily-Blant et al. 2013, 2017 ; Füri \& Marty 2015). The efficiency of chemical mass fractionation, which is the only process expected in cold and shielded gas (Terzieva \& Herbst 2000), has recently been called into question both theoretically (Roueff et al. 2015; Wirström \& Charnley 2018) and observationally by measuring $\mathrm{HCN} / \mathrm{HC}^{15} \mathrm{~N}$ in the L1498 pre-stellar core (Magalhães et al. 2018). The obtained value, $338 \pm 28$, agrees very well with the present-day elemental ratio of $\sim 330$ proposed in Paper I and thus supports the prediction that chemical fractionation is not an efficient process even in cold pre-stellar cores. Further credence was provided by subsequent direct measurements in $\mathrm{HC}_{3} \mathrm{~N}$ and $\mathrm{HC}_{5} \mathrm{~N}$ toward similar sources (Hily-Blant et al. 2018; Taniguchi \& Saito 2017). The concordance between observations and models may be only an apparent one, however, because sharp deviations are recurrently obtained for $\mathrm{N}_{2} \mathrm{H}^{+}$(Bizzocchi et al. 2013; Redaelli et al. 2018).

The other fractionation process, selective photodissociation of $\mathrm{N}_{2}$, which is expected in unshielded gas, has been investigated in the context of protoplanetary disks and molecular clouds (Heays et al. 2014; Visser et al. 2018; Furuya \& Aikawa 2018). The opacity of $\mathrm{N}^{15} \mathrm{~N}$ is far lower than that of $\mathrm{N}_{2}$, therefore more ${ }^{15} \mathrm{~N}$ atoms are released upon dissociation than ${ }^{14} \mathrm{~N}$. This leads to an atomic isotopic ratio that is lower than the bulk. Gas-phase and/or surface chemistry then propagates this deviation into other species. A correlation (positive or negative) between the UV flux and the ${ }^{14} \mathrm{~N} /{ }^{15} \mathrm{~N}$ is therefore expected in some species. For $\mathrm{HCN}$ in disks, the $\mathrm{HCN} / \mathrm{HC}^{15} \mathrm{~N}$ is predicted to increase with decreasing UV flux.

In this Letter, we aim at providing the requisite evidence for two distinct $\mathrm{N}$ reservoirs within the disk that orbits the nearby T Tauri star TW Hya through an analysis of the emission of HCN and isotopologs, based on archival ALMA data (2016.1.00629.S, Cleeves et al.). We also explore the possibility of a radial gradient in the $\mathrm{HCN} / \mathrm{HC}^{15} \mathrm{~N}$ to determine whether selective photodissociation is the driving fractionation process in PSN analogs.

\section{Observations and data reduction}

The (4-3) rotational transitions of $\mathrm{HCN}, \mathrm{H}^{13} \mathrm{CN}$, and $\mathrm{HC}^{15} \mathrm{~N}$ at $354.5,345.4$, and $344.2 \mathrm{GHz}$, respectively, have been mapped with the ALMA interferometer with a spectral resolution of $61 \mathrm{KHz}$ or $0.053 \mathrm{~km} \mathrm{~s}^{-1}$ (see Appendix A). All the lines were

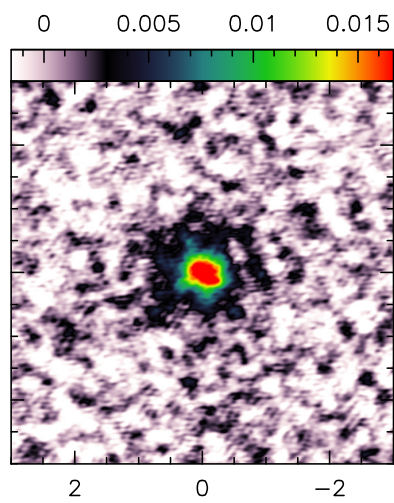

Fig. 1. Integrated intensity maps of $\mathrm{HCN}, \mathrm{H}^{13} \mathrm{CN}$, and $\mathrm{HC}^{15} \mathrm{~N}$ (from left to right) in Jy beam $\mathrm{km} \mathrm{s}^{-1}$. The maps are centered at $\alpha=11: 01: 51.81150$ $\delta=-34: 42: 17.2636$ (J2000.0). The visibilities were projected on the same spatial grid and convolved to a synthesized beam of HPBW $0.26^{\prime \prime} \times 0.21^{\prime \prime}$. The emission is centered on the systemic velocity of $2.83 \mathrm{~km} \mathrm{~s}^{-1}$ and is integrated over $5 \mathrm{~km} \mathrm{~s}^{-1}$ for $\mathrm{HCN}$ and $2 \mathrm{~km} \mathrm{~s}^{-1}$ for the less abundant isotopologs. observed simultaneously, thus mitigating cross-calibration biases. The standard pipeline of CASA 5.4.0 was used to generate the complex visibilities, which were then self-calibrated in Gildas $^{2}$. The final sensitivities are 2.3, 1.9, and $2.0 \mathrm{mJy}^{\text {beam }}{ }^{-1}$ per $61 \mathrm{kHz}$ channel for $\mathrm{HCN}, \mathrm{H}^{13} \mathrm{CN}$, and $\mathrm{HC}^{15} \mathrm{~N}$ respectively. The resulting integrated intensity maps are shown in Fig. 1.

The HCN(4-3) spectra (see Fig. 2) contain two weak hyperfine (hf) transitions of equal intensity, each carrying $2.1 \%$ of the total flux, and shifted by -1.672 and $+1.360 \mathrm{~km} \mathrm{~s}^{-1}$ with respect to our reference frequency $354505.477 \mathrm{MHz}$. They surround a strong, non-Gaussian, central feature made of three overlapping hf transitions representing $95.8 \%$ of the flux. The intrinsically weaker intensity of $\mathrm{H}^{13} \mathrm{CN}$ and $\mathrm{HC}^{15} \mathrm{~N}$ compared to $\mathrm{HCN}$ leads to one single feature only, which in the case of $\mathrm{H}^{13} \mathrm{CN}$ carries $95.8 \%$ of the total flux. For $\mathrm{HC}^{15} \mathrm{~N}$, the splitting is entirely unresolved and the single feature thus carries $100 \%$ of the rotational transition flux (see Table A.1).

\section{Results}

The conversion of line flux into abundance ratios is a simple procedure provided the lines are optically thin and the excitation temperatures are known. Even for optically thin transitions, however, the overlap in velocity space of lines that are emitted at different locations in the disk, which is a result of the Keplerian rotation of the disk, complicates the conversion from flux into abundance ratios because this mixes different regions of the disk, broadens the lines, and may also weaken the assumption that the emitting species are cospatial. The latter is especially true in the case of species with very different abundances, such as nitrogen isotopologs. Mitigating both the optical depth and Keplerian blurring effects thus offers clear advantages.

The nearly face-on configuration of the TW Hya disk naturally reduces the Keplerian blurring. In addition, the contribution from different locations along the line of sight to the emerging lines is limited to the vertical direction. Moreover, the spectrally resolved hf multiplet of $\mathrm{HCN}$ provides two weak lines that are most likely optically thin. Their flux is thus directly proportional to their column density.

Because the emission of all three isotopologs is well detected and spatially resolved, one goal might be measuring the $\mathrm{HCN}$ isotopic ratios as a function of radius, provided that line broadening can be minimized. This implies that the spatial area over which spectra are averaged needs to be limited. Another

2 IRAM memo available at http://www.iram.fr/IRAMFR/ARC/ documents/filler/casa-gildas.pdf 


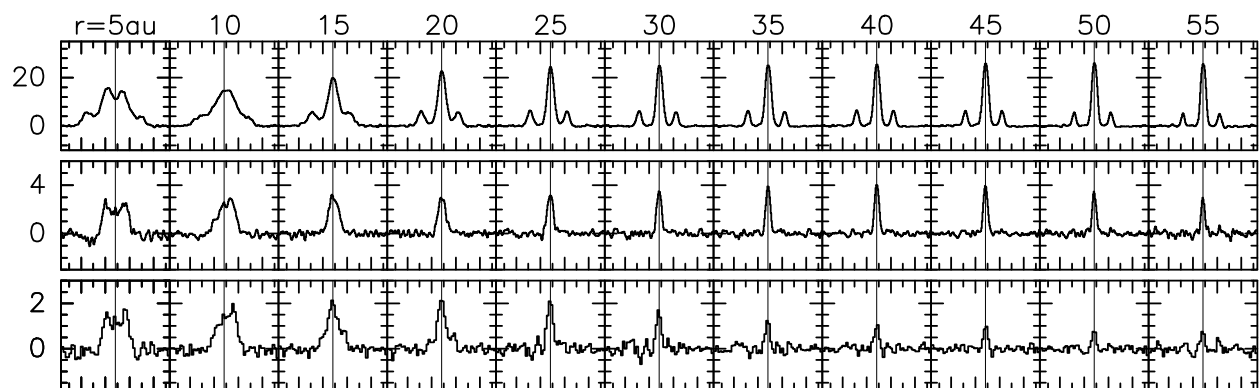

0246

Velocity $(\mathrm{km} / \mathrm{s})$
Fig. 2. Spectra of $\mathrm{HCN}, \mathrm{H}^{13} \mathrm{CN}$, and $\mathrm{HC}^{15} \mathrm{~N}$ (from top to bottom) from the Keplerian mask at $2.83 \mathrm{~km} \mathrm{~s}^{-1}$ (systemic velocity) and averaged within annuli centered at radii $r=5$ to $55 \mathrm{au}$. The specific intensity scale is $\mathrm{K}$ using $\mathrm{K} /\left(\mathrm{Jy}_{\text {beam }}{ }^{-1}\right)$ conversion factors of $178.7,188.1$, and 189.0 for $\mathrm{HCN}, \mathrm{H}^{13} \mathrm{CN}$, and $\mathrm{HC}^{15} \mathrm{~N}$, respectively.
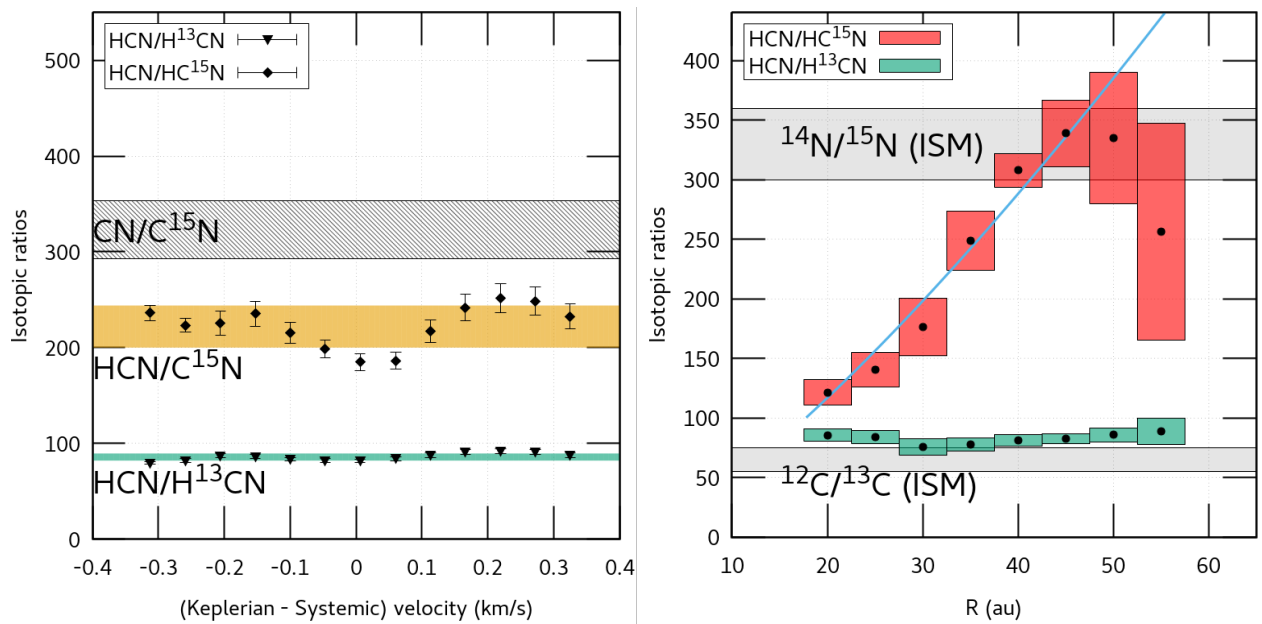

Fig. 3. Left: $\mathrm{HCN} / \mathrm{HC}^{15} \mathrm{~N}$ and $\mathrm{HCN} / \mathrm{H}^{13} \mathrm{CN}$ isotopic ratios in each Keplerian channel derived from the spatially averaged procedure (see Sect. 3.1). The colored bands indicate the average value $\pm 1 \sigma$. The spatially averaged $\mathrm{CN} / \mathrm{C}^{15} \mathrm{~N}$ ratio from Paper I is shown as a hatched rectangle. Right: radial dependence (see Sect. 3.2) of the $\mathrm{HCN} / \mathrm{H}^{13} \mathrm{CN}$ and $\mathrm{HCN} / \mathrm{HC}^{15} \mathrm{~N}$ ratios. Each box is 5 au wide (one-third of the $\mathrm{HPBW}$ ) and $\pm 1 \sigma$ in height. The full line shows the best fit of a power law to the ${ }^{15} \mathrm{~N}$ ratio, with a slope of 1.3 . The gray shaded areas are the present-day ${ }^{14} \mathrm{~N} /{ }^{15} \mathrm{~N}$ and ${ }^{12} \mathrm{C} /{ }^{13} \mathrm{C}$ elemental ratios in the local ISM (330 \pm 30 (Paper I) and $65 \pm 10$ Halfen et al. 2017, respectively).

complementary approach consists of deriving a spatially averaged isotopic ratio by measuring line flux ratios over the whole disk. This method provides higher sensitivity, but Keplerian line broadening must still be controlled. We applied both approaches and detail them in the following.

\subsection{Spatially averaged line fluxes}

In order to limit the Keplerian line-broadening, the spectra were averaged over several groups of pixels that were selected for their closely similar projected Keplerian velocity. In practice, a 2D mask was thus determined by selecting the disk area where the $354507.455 \mathrm{MHz}$ hyperfine emission of $\mathrm{HCN}$ is higher than $40 \mathrm{mJy}$ beam $^{-1}$ in each velocity channel. Although the overlap of different Keplerian velocities is well controlled, it is still noticeable close to the disk center, and we further masked out the spectra located at radii smaller than $0.3^{\prime \prime}$. The resulting mask recovered the usual dipole-like pattern, but with a central hole due to the radial filter (see Fig. B.1). As a result, the spectra averaged within each Keplerian velocity mask have a high signal-to-noise ratio (see Fig. B.2), allowing the two weak hf transitions of $\mathrm{HCN}$ to be properly fitted. The line fluxes of the two weak hf lines at 354503.869 and $354507.455 \mathrm{MHz}$ were measured through Gaussian fitting and their averaged value, corrected for their summed relative intensity $(4.2 \%)$, was then used as an estimator of the HCN flux (see Table B.1).

\subsection{Radially dependent fluxes}

In order to measure the flux ratios as a function of distance from the central $\mathrm{T}$ Tauri star and still avoid Keplerian linebroadening, a radial mask was superimposed on the previously described Keplerian masks. To calculate disk radial offsets in au, we adopted a distance of $59.5 \pm 0.9 \mathrm{pc}$ to TW Hya (Gaia Collaboration 2018). Spectra contained within concentric annuli, centered at radii $r=5-55$ au in steps of 5 au (one-third of the half-power beam width, HPBW), and of constant thickness $\delta r=5 \mathrm{au}$, were averaged. The outer radius was imposed by the $\mathrm{HC}^{15} \mathrm{~N}$ detection limit (see Fig. 1). The number of spectra in each annulus increased with $r$, which compensated for the natural decrease of the signal-to-noise ratio with radius. In the process, spectra were weighted according to their flux rms. As in the spatially averaged approach, the line fluxes of $\mathrm{HCN}, \mathrm{H}^{13} \mathrm{CN}$, and $\mathrm{HC}^{15} \mathrm{~N}$ were measured through Gaussian fitting.

Radially averaged spectra corresponding to the Keplerian mask at $2.83 \mathrm{~km} \mathrm{~s}^{-1}$ are shown in Fig. 2 . The velocity confusion at small radii is evident, while at radii above $20 \mathrm{au}$, it is efficiently removed by our masking procedure. Average spectra in all Keplerian channels are displayed in Fig. B.3. While the peak intensity of $\mathrm{HCN}$ hardly changes beyond $20 \mathrm{au}$, the peak intensities of $\mathrm{H}^{13} \mathrm{CN}$ and $\mathrm{HC}^{15} \mathrm{~N}$ decrease when moving outward. Also, all lines become narrower as the radius increases (see Table B.3). For HCN, the FWHM is $1.5 \pm 0.7 \mathrm{~km} \mathrm{~s}^{-1}$ at $r \leq 0.2^{\prime \prime}$, decreasing to $0.34 \pm 0.02 \mathrm{~km} \mathrm{~s}^{-1}$ at $0.9^{\prime \prime}$. 
Table 1. Isotopic ratios derived from our two approaches, the spatially averaged and the radially dependent methods (see also Fig. 3).

\begin{tabular}{rccc}
\hline \hline & $\mathrm{HCN} / \mathrm{HC}^{15} \mathrm{~N}$ & $\mathrm{HCN} / \mathrm{H}^{13} \mathrm{CN}$ & $\mathrm{H}^{13} \mathrm{CN} / \mathrm{HC}^{15} \mathrm{~N}$ \\
\hline Average & $223 \pm 21$ & $86 \pm 4$ & $2.6 \pm 0.2$ \\
\hline$r(\mathrm{au})=20$ & $121.4 \pm 10.6$ & $85.6 \pm 5.2$ & $1.4 \pm 0.1$ \\
25 & $140.4 \pm 14.6$ & $84.2 \pm 5.7$ & $1.7 \pm 0.1$ \\
30 & $176.4 \pm 24.1$ & $75.9 \pm 7.0$ & $2.4 \pm 0.3$ \\
35 & $248.7 \pm 24.6$ & $77.9 \pm 5.5$ & $3.2 \pm 0.4$ \\
40 & $307.8 \pm 14.2$ & $81.2 \pm 4.8$ & $3.7 \pm 0.3$ \\
45 & $338.7 \pm 28.2$ & $82.5 \pm 4.2$ & $4.2 \pm 0.4$ \\
50 & $334.9 \pm 55.0$ & $85.9 \pm 5.9$ & $3.9 \pm 0.7$ \\
55 & $256.4 \pm 91.2$ & $88.7 \pm 11.1$ & $2.9 \pm 1.1$ \\
\hline
\end{tabular}

\section{Isotopic ratios}

Because the three molecules have very similar spectroscopic constants, their rotational flux ratios are equal to their column density ratios to within $1 \%$, provided the lines are optically thin and have the same excitation temperature. Under these assumptions, which we verify below, we obtain the isotopic ratios directly from the fluxes. The results are shown in Fig. 3 and are listed in Tables 1 and B.2.

For $\mathrm{HCN} / \mathrm{HC}^{15} \mathrm{~N}$, we find ratios between 185 and 252 across the Keplerian channels (typical uncertainty of 5-10\%) with an average isotopic ratio $223 \pm 21$. Here and elsewhere, uncertainties on the absolute flux include a conservative $10 \%$ error (statistical) from amplitude calibration. The quoted uncertainty on average values is the largest of the weighted uncertainties and the dispersion of the averaged values. The lowest values (185, 186, and 198) are obtained in the three central Keplerian channels, and the average ratio in the eight remaining channels is $233 \pm 12$. $\mathrm{HCN} / \mathrm{H}^{13} \mathrm{CN}$ is more uniform, with values ranging from 82 to 92 , which averages to $86 \pm 4$. The corresponding average of $\mathrm{H}^{13} \mathrm{CN} / \mathrm{HC}^{15} \mathrm{~N}$ is $2.6 \pm 0.2$, with values ranging from 2.22 to 2.75 . The low values of $\mathrm{HCN} / \mathrm{HC}^{15} \mathrm{~N}$ might arise because the opacity of $\mathrm{HCN}$ is substantial even in its weak hf lines. However, the almost constant $\mathrm{HCN} / \mathrm{H}^{13} \mathrm{CN}$ suggests that these lines are instead optically thin. Still, assuming a constant isotopic ratio of 233 and adopting the usual expression $\tau /(1-\exp (-\tau))$ for the opacity correction for the integrated intensity, with $\tau$ the opacity (Goldsmith \& Langer 1999), an opacity of 0.4 for the weakest hf lines, corresponding to a rotational opacity $\tau_{12} \approx 20$, would be needed to explain the flux ratios in these three channels. Alternatively, assuming a constant $\mathrm{H}^{13} \mathrm{CN} / \mathrm{HC}^{15} \mathrm{~N}$ ratio of 2.7 based on the outer channels (see Table B.2), the total $\mathrm{H}^{13} \mathrm{CN}$ opacity would be $\tau_{13}=0.3$ in the three middle channels. It is thus likely that the opacity of the weakest hf line of $\mathrm{HCN}(4-3)$ and of $\mathrm{H}^{13} \mathrm{CN}(4-3)$ is $\approx 0.3$, which is not entirely negligible, but still fulfills our optically thin assumption. We note that in these three channels, the $\mathrm{HCN} / \mathrm{H}^{13} \mathrm{CN}$ would change by less than $4 \%$ if these corrections were applied.

The radially dependent approach shows that the HCN-to$\mathrm{HC}^{15} \mathrm{~N}$ isotopic ratio varies with radius while $\mathrm{HCN} / \mathrm{H}^{13} \mathrm{CN}$ remains constant within $1 \sigma$. More specifically, the former ratio increases from 121 at 20 au to 339 at $45 \mathrm{au}$, and may decrease at larger distances. The nitrogen isotopic ratio in HCN close to the star is thus a factor 2.7 lower than the spatially averaged $\mathrm{CN} / \mathrm{C}^{15} \mathrm{~N}, 323 \pm 30$, whereas between 45 and $50 \mathrm{au}$, the two ratios become consistent within $1 \sigma$. The ratios change by less than $4 \%$ when the three central Keplerian channels are excluded. The best fit of a power law to the $\mathrm{HCN} / \mathrm{HC}^{15} \mathrm{~N}$ gives $117 \pm 10 \times(r / 20 \mathrm{au})^{1.3 \pm 0.13}$, indicating a shallower dependence with radius than a pure geometrical dilution of the UV flux from the central star $\left(\propto r^{-2}\right)$.

A key assumption in our analysis is that a single excitation temperature characterizes the weak hf lines of $\mathrm{HCN}$ and the overlapping hf lines of $\mathrm{H}^{13} \mathrm{CN}$ and of $\mathrm{HC}^{15} \mathrm{~N}$. However, line overlap may lead to unequal excitation temperatures among the hf manifold of HCN (Magalhães et al. 2018). We have tested our assumption (see Appendix C) by computing the excitation temperatures of $\mathrm{HCN}, \mathrm{H}^{13} \mathrm{CN}$, and $\mathrm{HC}^{15} \mathrm{~N}$ lines while taking line overlap in the excitation into account. Our calculations assume uniform physical conditions and cover the density and gas temperature regimes from $10^{6}$ to $10^{11} \mathrm{~cm}^{-3}$ and 5 to $30 \mathrm{~K}$, respectively. We found that deviations from a single excitation temperature are only obtained at densities below $3 \times 10^{7} \mathrm{~cm}^{-3}$ (see Fig. C.1). On the other hand, the full width at half-maximum (FWHM) of the $\mathrm{HCN}, \mathrm{H}^{13} \mathrm{CN}$, and $\mathrm{HC}^{15} \mathrm{~N}$ lines at a radius of $0.6^{\prime \prime}(\approx 35 \mathrm{au})$ and beyond is $\approx 0.35 \mathrm{~km} \mathrm{~s}^{-1}$, which places an upper limit on the kinetic temperature of $(F W H M / 2.35)^{2} \times \mu m_{\mathrm{H}} /\left(2 k_{\mathrm{B}}\right) \approx 35 \mathrm{~K}$ (Teague et al. 2016). Referring to the TW Hya model of van't Hoff et al. (2017), this upper limit indicates that the density is at these radii higher than $10^{9} \mathrm{~cm}^{-3}$, which means that it is in a regime where lines are expected to be thermalized. This validates our basic assumption. Our upper limit on the kinetic temperature is also well below the gas temperature of $\sim 200 \mathrm{~K}$ where the HCN abundance is the highest in the generic models of Visser et al. (2018).

\section{Discussion}

The spatially averaged directly measured $\mathrm{CN} / \mathrm{C}^{15} \mathrm{~N}$ and $\mathrm{HCN} / \mathrm{HC}^{15} \mathrm{~N}$ in the TW Hya circumstellar disk provide clearcut confirmation of at least two isotopic reservoirs of nitrogen, as anticipated by Paper I. In CN, the ratio is $1.4 \pm 0.2$ times higher than in HCN, and their difference is $90 \pm 36$. Based on the $\sim 8 \mathrm{Myr}$ age of TW Hya, these results indicate that multiple isotopic reservoirs are present at the key protoplanetary disk evolutionary stages when comets and giant planets are likely forming.

In the comet formation zone between 20 and $30 \mathrm{au}$, the $\mathrm{HCN} / \mathrm{HC}^{15} \mathrm{~N}$ is $121 \pm 11$, which is a factor of 2.8 compared to the outer disk at $55 \mathrm{au}$, where the isotopic ratio of $\mathrm{HCN}$ matches the revised elemental ratio of 330 well. The ${ }^{15} \mathrm{~N}$ enrichment in the region inside of $30 \mathrm{au}$ is reminiscent of the threefold ${ }^{15} \mathrm{~N}$-enrichment of comets compared to the bulk protoSun, providing a strong indication that $\mathrm{HCN}$ traces the fractionated reservoir that is recorded by comets. Guzmán et al. (2017) also found marginal evidence for an increase of the HCN-based ${ }^{14} \mathrm{~N} /{ }^{15} \mathrm{~N}$ ratio with radius in the inner $\sim 50$ au of the V4046Sgr disk. We also note that the inner disk ratio confirms the mean $\mathrm{HCN} / \mathrm{HC}^{15} \mathrm{~N}$ ratio of $111 \pm 19$ (Paper I) that was obtained indirectly by Guzmán et al. (2017) assuming $\mathrm{HCN} / \mathrm{H}^{13} \mathrm{CN}=70 \pm 20$. Adopting our measured averaged of 86 for the latter would bring the former ratio to $136 \pm 23$. We also very tentatively observe a decrease of the ratio beyond $55 \mathrm{au}$, which could indicate chemical processes in a gas not exposed to strong UV radiation and where depletion is limited. Measurements with a high signal-to-noise ratio beyond 55 au are needed to confirm this behavior, however.

The average $\mathrm{HCN} / \mathrm{H}^{13} \mathrm{CN}, 86 \pm 4$, is significantly higher than the ${ }^{12} \mathrm{C} /{ }^{13} \mathrm{C}$ ratio of $65 \pm 10$ in the local ISM (Halfen et al. 2017), but agrees surprisingly well with the value of $89 \pm 1$ in the PSN (Clayton \& Nittler 2004). This indicates that HCN is slightly depleted in ${ }^{13} \mathrm{C}$ in TW Hya, opposite to what was found in the L1498 pre-stellar core (Magalhães et al. 2018), suggesting that 
the underlying process may not be the same in these two environments. The depletion of $\mathrm{HCN}$ in ${ }^{13} \mathrm{C}$ is at variance with the models of Visser et al. (2018), however, which instead predict that $\mathrm{HCN}$ is enriched in ${ }^{13} \mathrm{C}$ at radii below $\sim 100$ au. Optically thick $\mathrm{H}^{13} \mathrm{CN}$ emission could artificially increase the $\mathrm{HCN} / \mathrm{H}^{13} \mathrm{CN}$ provided that the weak $\mathrm{HCN}$ hf transition remains thin. However, the $\mathrm{H}^{13} \mathrm{CN}$ opacity required to decrease the ratio to the elemental value is 0.55 , which is higher than the value of 0.3 derived previously for the central Keplerian channels. This also implies that the total opacity of HCN would be 35 , or 0.75 for the weakest $\mathrm{HCN}$ hf, which is twice higher than the value of 0.3 that is required to bring the spatially averaged $\mathrm{HCN} / \mathrm{HC}^{15} \mathrm{~N}$ to a constant value (see Sect. 4). We note that high ${ }^{12} \mathrm{C} /{ }^{13} \mathrm{C}$ ratios have already been measured in carbon monoxide from ices toward young stellar objects (Smith et al. 2015).

The increasing $\mathrm{HCN} / \mathrm{HC}^{15} \mathrm{~N}$ with radius may indicate that selective photodissociation represents the dominant fractionation process in disks (Visser et al. 2018). In these models, the $\mathrm{HCN}$-to-HC ${ }^{15} \mathrm{~N}$ column density ratio first decreases with radius before it increases back to the elemental ratio as a consequence of UV flux attenuation that is due to a combination of geometrical dilution and dust extinction. We note that the minimum ratio in the grid of models of Visser et al. (2018) is $\approx 240-280$ for disks around T Tauri stars, which would translate into 180-210 when 330 (rather than 441) is assumed for the elemental ratio. This is still higher than the lowest ratio reported here and might indicate that the UV flux is underestimated in these models. However, dedicated models of TW Hya are required because the precise radial dependence of the isotopic ratio strongly depends on the physical parameters of the model (disk mass, grain size, and dust-to-gas mass ratio).

Overall, the increasing $\mathrm{HCN} / \mathrm{HC}^{15} \mathrm{~N}$ from 121 up to the bulk value in the outer disk supports a scenario in which a single reservoir of interstellar, not fractionated, nitrogen dominates at large radii in PSN analogs. This nitrogen reservoir is enriched in ${ }^{15} \mathrm{~N}$ by the selective photodissociation of $\mathrm{N}_{2}$ at smaller distance from the central star.

Acknowledgements. PHB thanks D. Petry for his help in processing the ALMA data, and A. Faure for useful comments and discussions. We also thank the referee for valuable comments that greatly clarified the paper. This study makes use of the ADS/JAO.ALMA\#2016.1.00629.S data. ALMA is a partnership of ESO (representing its member states), NSF (USA) and NINS (Japan), together with NRC (Canada), MOST and ASIAA (Taiwan), and KASI (Republic of Korea), in cooperation with the Republic of Chile. The Joint ALMA Observatory is operated by ESO, AUI/NRAO and NAOJ. This work has received financial support from the Institut Universitaire de France and from Labex OSUG@2020. JHK's research on protoplanetary disks orbiting young stars near Earth is supported by NASA Exoplanets Research Program grants NNX16AB43G and 80NSSC19K0292 to RIT. This study made use of the CDMS database (Müller et al. 2005)

\section{References}

Beckwith, S. V. W., \& Sargent, A. I. 1993, ApJ, 402, 280

Bizzocchi, L., Caselli, P., Leonardo, E., \& Dore, L. 2013, A\&A, 555, A109

Bonal, L., Huss, G. R., Krot, A. N., et al. 2010, Geochim. Cosmochim. Acta, 74 6590

Clayton, D. D., \& Nittler, L. R. 2004, ARA\&A, 42, 39

Daniel, F., \& Cernicharo, J. 2008, A\&A, 488, 1237

Fouchet, T., Lellouch, E., Bézard, B., et al. 2000, Icarus, 143, 223

Füri, E., \& Marty, B. 2015, Nat. Geosc., 8, 515

Furuya, K., \& Aikawa, Y. 2018, ApJ, 857, 105

Gaia Collaboration (Brown, A. G. A., et al.) 2018, A\&A, 616, A1

Goldsmith, P. F., \& Langer, W. D. 1999, ApJ, 517, 209

Guzmán, V. V., Öberg, K. I., Huang, J., Loomis, R., \& Qi, C. 2017, ApJ, 836, 30

Halfen, D. T., Woolf, N. J., \& Ziurys, L. M. 2017, ApJ, 845, 158

Heays, A. N., Visser, R., Gredel, R., et al. 2014, A\&A, 562, A61

Hily-Blant, P., Bonal, L., Faure, A., \& Quirico, E. 2013, Icarus, 223, 582

Hily-Blant, P., Magalhaes, V., Kastner, J., et al. 2017, A\&A, 603, L6

Hily-Blant, P., Faure, A., Vastel, C., et al. 2018, MNRAS, 480, 1174

Magalhães, V. S., Hily-Blant, P., Faure, A., Hernandez-Vera, M., \& Lique, F. 2018, A\&A, 615, A52

Marty, B., Chaussidon, M., Wiens, R. C., Jurewicz, A. J. G., \& Burnett, D. S. 2011, Science, 332, 1533

Müller, H. S. P., Schlöder, F., Stutzki, J., \& Winnewisser, G. 2005, J. Mol. Struct. 742,215

Redaelli, E., Bizzocchi, L., Caselli, P., et al. 2018, A\&A, 617, A7

Romano, D., Matteucci, F., Zhang, Z.-Y., Papadopoulos, P. P., \& Ivison, R. J. 2017, MNRAS, 470, 401

Roueff, E., Loison, J. C., \& Hickson, K. M. 2015, A\&A, 576, A99

Smith, R. L., Pontoppidan, K. M., Young, E. D., \& Morris, M. R. 2015, ApJ, 813,120

Taniguchi, K. \& Saito, M. 2017, PASJ, 69, L7

Teague, R., Guilloteau, S., Semenov, D., et al. 2016, A\&A, 592, A49

Terzieva, R., \& Herbst, E. 2000, MNRAS, 317, 563

van't Hoff, M. L. R., Walsh, C., Kama, M., Facchini, S., \& van Dishoeck, E. F. 2017, A\&A, 599, A101

Visser, R., Bruderer, S., Cazzoletti, P., et al. 2018, A\&A, 615, A75

Wampfler, S., Rubin, M., Altwegg, K., et al. 2018, in COSPAR Meeting, 42nd COSPAR Scientific Assembly, 42, B1.3-21-18

Wirström, E. S., \& Charnley, S. B. 2018, MNRAS, 474, 3720 


\section{Appendix A: Observations}

The observations were performed with the ALMA interferometer (2016.1.00629.S, PI:I. Cleeves) and were spread over six epochs (2016 December 30, 2017 July 5, 2017 July 10, 2017 July 15, 2017 July 21, and 2017 July 22). J1037-2934 was used as a bandpass, flux, and phase calibrator. The phase was also calibrated with J1103-3251, and J1058+0133 was also used for bandpass calibration.

$\mathrm{H}^{13} \mathrm{CN}$ and $\mathrm{HC}^{15} \mathrm{~N}$ were tuned in the lower side-band and $\mathrm{HCN}$ in the upper side-band, allowing simultaneous observations for the three species. We used the standard pipeline of CASA 5.4.0 to generate the first sets of complex visibilities, which were then exported into the Gildas format using the Gildas-Casa filer of Guilloteau, Chapillon et al. (see the IRAM memo $^{3}$ ). Self-calibration was then applied to generate the final set of visibilities.

Spectroscopy shows that the three hyperfine (hf) transitions of $\mathrm{HCN}(4-3)$ are separated by less than $0.13 \mathrm{~km} \mathrm{~s}^{-1}$ and are not resolved. Their total relative intensity is $95.8 \%$. The hf transition at $354505.846 \mathrm{MHz}$ is not detected given its $0.03 \%$ relative strength. The properties of the hf lines of $\mathrm{HCN}$ and $\mathrm{H}^{13} \mathrm{CN}$ are summarized in Table A.1, while for $\mathrm{HC}^{15} \mathrm{~N}$, the unresolved hyperfine splitting is not given. We also show in Fig. B.1 channel maps of $\mathrm{HCN}$ strong central feature, $\mathrm{H}^{13} \mathrm{CN}$, and $\mathrm{HC}^{15} \mathrm{~N}$. The velocity range covers the main and overlapping hf lines of HCN.
Table A.1. Spectroscopic information on the observed transitions.

\begin{tabular}{lcccc}
\hline $\begin{array}{l}\text { Rest freq. }{ }^{(a)} \\
\text { MHz }\end{array}$ & $\begin{array}{c}\delta v^{(b)} \\
\mathrm{km} \mathrm{s}^{-1}\end{array}$ & $\begin{array}{r}A_{\mathrm{ul}}{ }^{(c)} \\
\mathrm{s}^{-1}\end{array}$ & $g_{u}{ }^{(d)}$ & R. I. $^{(e)}$ \\
\hline & & $\mathrm{HCN}$ & & \\
354503.869 & 1.360 & $1.284(-4)$ & 9 & $2.083(-2)$ \\
354505.367 & 0.094 & $1.886(-3)$ & 7 & $2.381(-1)$ \\
354505.477 & 0.000 & $1.925(-3)$ & 9 & $3.125(-1)$ \\
354505.523 & -0.039 & $2.054(-3)$ & 11 & $4.075(-1)$ \\
354505.846 & -0.312 & $2.620(-6)$ & 7 & $3.307(-4)$ \\
354507.455 & -1.672 & $1.650(-4)$ & 7 & $2.083(-2)$ \\
& & $\mathrm{H}^{13} \mathrm{CN}$ & & \\
345338.160 & -1.398 & $1.190(-4)$ & 9 & $2.089(-2)$ \\
345339.660 & -0.095 & $1.740(-3)$ & 7 & $2.376(-1)$ \\
345339.770 & 0.000 & $1.780(-3)$ & 9 & $3.125(-1)$ \\
345339.810 & 0.035 & $1.900(-3)$ & 11 & $4.077(-1)$ \\
345340.140 & 0.321 & $2.420(-6)$ & 7 & $3.305(-4)$ \\
345341.750 & 1.719 & $1.530(-4)$ & 7 & $2.089(-2)$ \\
& & $\mathrm{HC} \mathrm{C}^{15} \mathrm{~N}$ & & \\
344200.109 & 0.000 & $1.8793(-3)$ & 9 & 1 \\
\hline
\end{tabular}

Notes. Numbers are written in the form $a(b)=a \times 10^{b} .{ }^{(a)}$ Rest frequency. ${ }^{(b)} \delta v$ is the velocity shift of each hf transition within a given multiplet. ${ }^{(c)}$ Coefficient for spontaneous decay. ${ }^{(d)}$ Upper level total degeneracy. ${ }^{(e)}$ The relative intensity of each hf line, normalized to a sum of 1 .

\section{Appendix B: Keplerian masks}

\section{B.1. Results from the spatially averaged procedure}

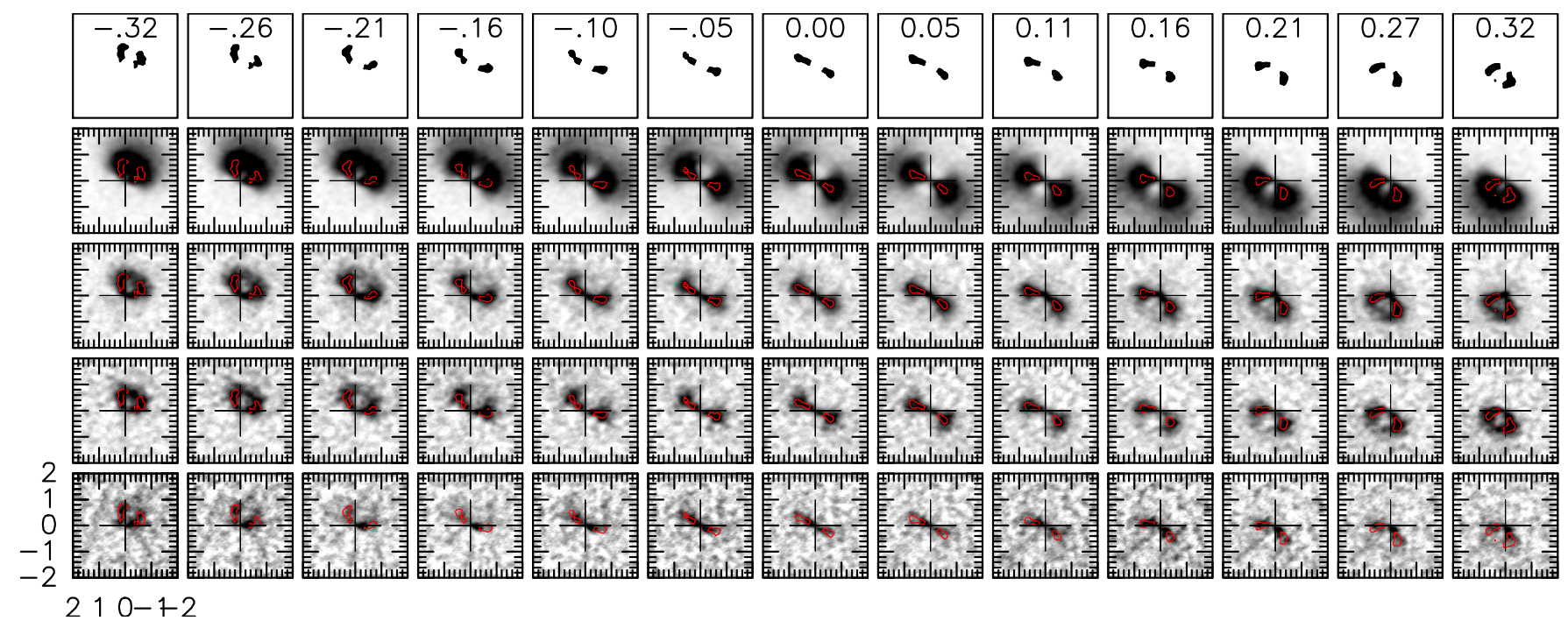

Fig. B.1. Keplerian masks (top row) overlaid on (from top to bottom) the $\mathrm{HCN}$ (main feature and weak hf line at $345507.455 \mathrm{MHz}$ ), $\mathrm{H}^{13} \mathrm{CN}$, and $\mathrm{HC}^{15} \mathrm{~N}$, channel maps. The masks (top row) were obtained for a threshold of $40 \mathrm{mJy} \mathrm{beam}^{-1}$ applied to the weak hf transition at $354507.455 \mathrm{MHz}$.

\footnotetext{
3 http://www.iram.fr/IRAMFR/ARC/documents/filler/ casa-gildas.pdf
} 


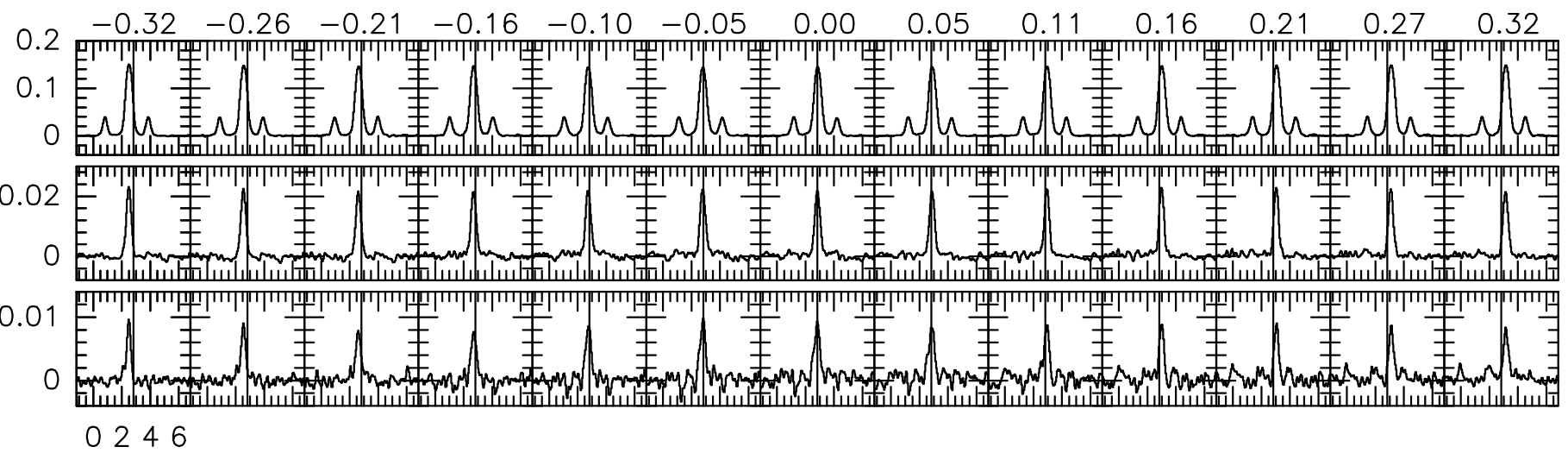

Velocity $(\mathrm{km} / \mathrm{s})$

Fig. B.2. Spatially averaged spectra $\left(\mathrm{Jy}_{\text {beam }}{ }^{-1}\right.$ ) within each Keplerian velocity channel. $\mathrm{HCN}, \mathrm{H}^{13} \mathrm{CN}$, and $\mathrm{HC}^{15} \mathrm{~N}$ are shown from top to bottom . The Keplerian channels are those of Fig. B.1.

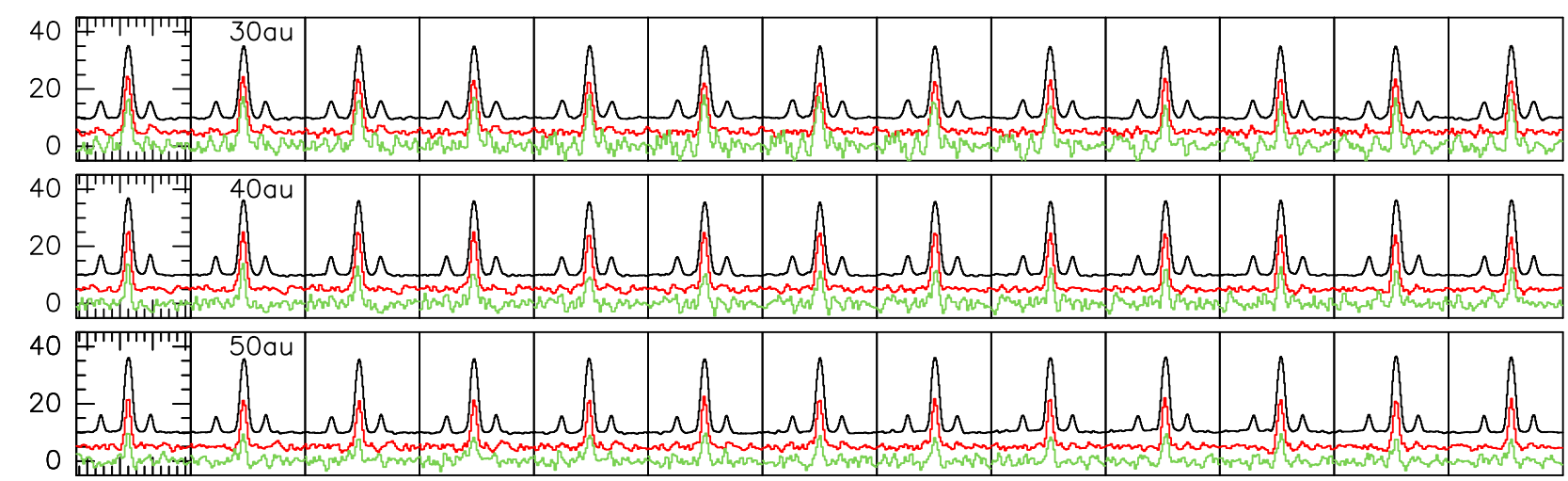

0246

Velocity $(\mathrm{km} / \mathrm{s})$

Fig. B.3. Spectra of $\mathrm{HCN}$ (black), $\mathrm{H}^{13} \mathrm{CN}(\mathrm{red}, \times 5)$, and $\mathrm{HC}^{15} \mathrm{~N}$ (green, $\left.\times 10\right)$ averaged within each Keplerian velocity channel (see, e.g., Table B.1) and within annuli of constant thickness 5 au, centered at 30, 40, and 50 au (from top to bottom). The specific intensity scale is $\mathrm{K}_{\text {using }} \mathrm{K} /\left(\mathrm{Jy}\right.$ beam ${ }^{-1}$ ) conversion factors of $178.7,188.1$, and 189.0 for $\mathrm{HCN}, \mathrm{H}^{13} \mathrm{CN}$, and $\mathrm{HC}^{15} \mathrm{~N}$, respectively. The spectra are shifted vertically for the sake of legibility.

In Fig. B.1 (top row), we show the Keplerian mask defined as explained in the main text. Each mask is overlaid in the channel maps of the three isotopologs, showing the usual dipole-like pattern (Beckwith \& Sargent 1993). Comparing the rows showing the main feature and the weak hf line of $\mathrm{HCN}$, we note that the extended $\mathrm{HCN}$ emission is filtered out in the present study, as is the case of $\mathrm{H}^{13} \mathrm{CN}$, although to a lesser extent. The spectra averaged within each mask, shown in Fig. B.2, were fit with Gaussian profiles, the results of which are summarized in Table B.1. The obtained fluxes are then used to derive the spatially averaged isotopic ratios listed in Table B.2.

\section{B.2. Radial dependence procedure}

By combining two masks, one in velocity space (Keplerian mask) and one in the radial direction, we are able to study the radial dependence of the ${ }^{14} \mathrm{~N} /{ }^{15} \mathrm{~N}$ isotopic ratio in $\mathrm{HCN}$ toward the TW Hya system. In Fig. 2, we show the spectra averaged within each annulus, but we concentrate on the central Keplerian channel at $2.83 \mathrm{~km} \mathrm{~s}^{-1}$ (systemic velocity). Figure B. 3 is complementary in that it shows the spectra averaged at three radii, but within all Keplerian masks.

As in the radially averaged approach, the spectral line intensity and shape at any radius do not change appreciably in the various Keplerian velocity channels. In contrast, the dependence on radius is significant, especially for $\mathrm{H}^{13} \mathrm{CN}$ and most importantly for $\mathrm{HC}^{15} \mathrm{~N}$. As can be seen in Table B.3 and as mentioned in the main text, all lines become narrower as the radius increases (see Table B.3).

\section{B.3. Radially dependent line width}

Gaussian fits to Keplerian-filtered spectra averaged within concentric annuli of thickness $0.1^{\prime \prime}$ were performed in order to measure the dependence of the FWHM of the $\mathrm{HCN}, \mathrm{H}^{13} \mathrm{CN}$, and $\mathrm{HC}^{15} \mathrm{~N}$ lines. The results are summarized in Table B.3. They are used to infer an upper limit on the kinetic temperature following Paper I and Teague et al. (2016). 
Table B.1. Results of the Gaussian fits to the spatially averaged spectra of Fig. B.2.

\begin{tabular}{|c|c|c|c|c|c|c|}
\hline \multirow[t]{2}{*}{$v_{K}-v_{0}$} & \multicolumn{2}{|c|}{$\mathrm{HCN}^{(a)}$} & \multicolumn{2}{|c|}{$\mathrm{H}^{13} \mathrm{CN}^{(b)}$} & \multicolumn{2}{|c|}{$\mathrm{HC}^{15} \mathrm{~N}^{(c)}$} \\
\hline & $W^{(\S)}$ & $v^{(\S)}$ & $W^{(\S)}$ & $v^{(\S)}$ & $W^{(\S)}$ & $v^{(\S)}$ \\
\hline-0.32 & $779.2(12.0)$ & $2.515(1)$ & $9.8(1)$ & 2.494(3) & $3.3(1)$ & 2.491(5) \\
\hline-0.26 & 803.2(13.1) & $2.568(1)$ & $9.8(2)$ & $2.541(3)$ & $3.6(1)$ & $2.536(7)$ \\
\hline-0.21 & $835.1(13.9)$ & $2.634(1)$ & $9.6(2)$ & $2.612(4)$ & $3.7(2)$ & $2.611(10)$ \\
\hline-0.16 & $871.0(12.7)$ & $2.707(1)$ & $10.1(2)$ & $2.698(3)$ & $3.7(2)$ & $2.711(11)$ \\
\hline-0.10 & $884.6(12.2)$ & $2.753(1)$ & $10.6(2)$ & $2.746(4)$ & $4.1(2)$ & $2.756(10)$ \\
\hline-0.05 & $892.4(13.7)$ & $2.799(1)$ & $10.9(2)$ & $2.788(5)$ & $4.5(2)$ & $2.784(11)$ \\
\hline 0.00 & $850.0(14.2)$ & $2.856(1)$ & $10.4(2)$ & $2.834(4)$ & $4.6(2)$ & $2.806(11)$ \\
\hline 0.05 & $838.8(14.1)$ & $2.899(1)$ & $10.0(2)$ & $2.877(4)$ & $4.5(2)$ & $2.840(12)$ \\
\hline 0.11 & 847.7(14.7) & $2.956(1)$ & $9.7(2)$ & $2.937(4)$ & $3.9(2)$ & $2.921(12)$ \\
\hline 0.16 & $870.9(16.9)$ & $3.010(1)$ & $9.6(2)$ & $2.988(4)$ & $3.6(2)$ & $2.990(10)$ \\
\hline 0.21 & $881.2(16.1)$ & $3.067(1)$ & $9.6(2)$ & $3.035(3)$ & $3.5(2)$ & $3.048(9)$ \\
\hline 0.27 & $870.2(15.5)$ & $3.117(1)$ & $9.6(2)$ & $3.080(4)$ & $3.5(2)$ & $3.113(10)$ \\
\hline 0.32 & $859.7(14.2)$ & $3.166(1)$ & $9.8(2)$ & $3.138(3)$ & $3.7(2)$ & $3.165(11)$ \\
\hline
\end{tabular}

Notes. Statistical uncertainties (at the $1 \sigma$ level) are written in parentheses in units of the last digit. $\left.{ }^{(}\right)$Keplerian minus systemic velocity $\left(\mathrm{km} \mathrm{s}^{-1}\right), W$ is the integrated flux in mJy beam ${ }^{-1} \mathrm{~km} \mathrm{~s}^{-1}$, and $v$ the central velocity in $\mathrm{km} \mathrm{s}^{-1}$. ${ }^{(a)}$ For HCN, the integrated intensity is computed as the total flux of the two weak lines divided by the sum of their relative intensities, $4.2 \%$, while the center velocity is that of the main central component. ${ }^{(b),(c)}$ The $\mathrm{hf}$ lines are not separated, and a single Gaussian was fit to the main line.

Table B.2. Isotopic ratios from the spatially averaged spectra.

\begin{tabular}{|c|c|c|c|c|c|c|c|c|c|c|c|c|}
\hline \multirow{2}{*}{$\frac{v_{K}-v_{0}{ }^{(\S)}}{-0.32}$} & \multicolumn{2}{|c|}{$W_{12}{ }^{(\dagger)}$} & \multicolumn{2}{|c|}{$W_{13}{ }^{(\dagger)}$} & \multicolumn{2}{|c|}{$W_{15}{ }^{(\dagger)}$} & \multicolumn{2}{|c|}{ 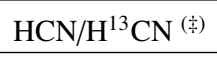 } & \multicolumn{2}{|c|}{$\mathrm{HCN} / \mathrm{HC}^{15} \mathrm{~N}^{(\ddagger)}$} & \multicolumn{2}{|c|}{$\mathrm{H}^{13} \mathrm{CN} / \mathrm{HC}^{15} \mathrm{~N}^{(\grave{\ddagger})}$} \\
\hline & 779.2 & 12.0 & 9.8 & 0.1 & 3.3 & 0.1 & 79.5 & 1.5 & 236.1 & 8.0 & 2.97 & 0.09 \\
\hline-0.26 & 803.2 & 13.1 & 9.8 & 0.2 & 3.6 & 0.1 & 82.0 & 2.1 & 223.1 & 7.2 & 2.72 & 0.09 \\
\hline-0.21 & 835.1 & 13.9 & 9.6 & 0.2 & 3.7 & 0.2 & 87.0 & 2.3 & 225.7 & 12.8 & 2.59 & 0.15 \\
\hline-0.16 & 871.0 & 12.7 & 10.1 & 0.2 & 3.7 & 0.2 & 86.2 & 2.1 & 235.4 & 13.2 & 2.73 & 0.16 \\
\hline-0.10 & 884.6 & 12.2 & 10.6 & 0.2 & 4.1 & 0.2 & 83.5 & 2.0 & 215.8 & 10.9 & 2.59 & 0.14 \\
\hline-0.05 & 892.4 & 13.7 & 10.9 & 0.2 & 4.5 & 0.2 & 81.9 & 2.0 & 198.3 & 9.3 & 2.42 & 0.12 \\
\hline 0.00 & 850.0 & 14.2 & 10.4 & 0.2 & 4.6 & 0.2 & 81.7 & 2.1 & 184.8 & 8.6 & 2.26 & 0.11 \\
\hline 0.05 & 838.8 & 14.1 & 10.0 & 0.2 & 4.5 & 0.2 & 83.9 & 2.2 & 186.4 & 8.9 & 2.22 & 0.11 \\
\hline 0.10 & 847.7 & 14.7 & 9.7 & 0.2 & 3.9 & 0.2 & 87.4 & 2.4 & 217.4 & 11.8 & 2.49 & 0.14 \\
\hline 0.16 & 870.9 & 16.9 & 9.6 & 0.2 & 3.6 & 0.2 & 90.7 & 2.6 & 241.9 & 14.2 & 2.67 & 0.16 \\
\hline 0.21 & 881.2 & 16.1 & 9.6 & 0.2 & 3.5 & 0.2 & 91.8 & 2.5 & 251.8 & 15.1 & 2.74 & 0.17 \\
\hline 0.27 & 870.2 & 15.5 & 9.6 & 0.2 & 3.5 & 0.2 & 90.6 & 2.5 & 248.6 & 14.9 & 2.74 & 0.17 \\
\hline 0.32 & 859.7 & 14.2 & 9.8 & 0.2 & 3.7 & 0.2 & 87.7 & 2.3 & 232.4 & 13.1 & 2.65 & 0.15 \\
\hline
\end{tabular}

Notes. ${ }^{(\S)}$ Keplerian minus systemic velocity $\left(\mathrm{km} \mathrm{s}^{-1}\right)$ is that of the hf line of $\mathrm{HCN}$ at $354507.455 \mathrm{MHz} .{ }^{(\dagger)} W_{12}, W_{13}$, and $W_{15}$ are

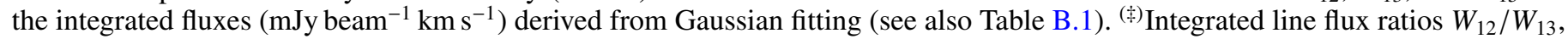
$W_{12} / W_{15}$, and $W_{13} / W_{15}$. All uncertainties are statistical and at the $1 \sigma$ level.

Table B.3. Radial dependence of the line width (FWHM, $\mathrm{km} \mathrm{s}^{-1}$ ) of $\mathrm{HCN}, \mathrm{H}^{13} \mathrm{CN}$, and $\mathrm{HC}^{15} \mathrm{~N}$.

\begin{tabular}{cccc}
\hline \hline$r\left({ }^{\prime \prime}\right)$ & $\mathrm{HCN}$ & $\mathrm{H}^{13} \mathrm{CN}$ & $\mathrm{HC}^{15} \mathrm{~N}$ \\
\hline 0.3 & $0.954 \pm 0.183$ & $0.832 \pm 0.101$ & $1.231 \pm 0.140$ \\
0.4 & $0.614 \pm 0.060$ & $0.603 \pm 0.038$ & $0.687 \pm 0.043$ \\
0.5 & $0.461 \pm 0.020$ & $0.458 \pm 0.034$ & $0.479 \pm 0.071$ \\
0.6 & $0.389 \pm 0.018$ & $0.382 \pm 0.020$ & $0.355 \pm 0.036$ \\
0.7 & $0.370 \pm 0.013$ & $0.369 \pm 0.020$ & $0.316 \pm 0.048$ \\
0.8 & $0.354 \pm 0.014$ & $0.371 \pm 0.016$ & $0.334 \pm 0.053$ \\
0.9 & $0.345 \pm 0.016$ & $0.367 \pm 0.030$ & $0.354 \pm 0.058$ \\
\hline
\end{tabular}

Notes. Spectra were averaged in annuli of thickness $0.1^{\prime \prime} . r$ is the outer radius of each annulus.

\section{Appendix C: Single excitation temperature assumption}

The main assumption in our analysis is that of a single excitation temperature characterizing the weak hf lines of $\mathrm{HCN}$ and the overlapping hf lines of $\mathrm{H}^{13} \mathrm{CN}$ and of $\mathrm{HC}^{15} \mathrm{~N}$. A thorough investigation of this assumption can only be made with twodimensional radiative transfer taking into account the overlap of hf transitions in the level population evaluation, which in turn requires a complete physical and kinematic structure of the disk. This type of analysis has been made in the context of pre-stellar starless cores by Magalhães et al. (2018), in spherical geometry, with the 1D ALICO model (Daniel \& Cernicharo 2008). 


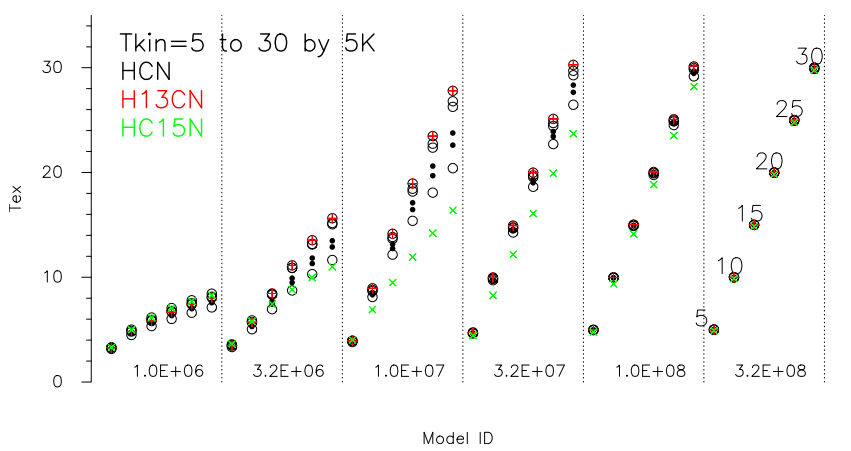

Fig. C.1. Excitation temperature of the six hf lines of HCN (see Table A.1) and the strongest hf lines of $\mathrm{H}^{13} \mathrm{CN}$ (red) and $\mathrm{HC}^{15} \mathrm{~N}$ (green). The four overlapping hf lines of $\mathrm{HCN}$ are shown as open circles and the two weakest resolved lines with filled dots.

In the present context, we were unable to make detailed calculations like this. Instead, we used our 1D ALICO code to investigate the density and kinetic gas temperature at which departures from the single excitation temperature assumption may be obtained. To do so, the level populations were computed while treating the effect of hf line overlap in the excitation of $\mathrm{HCN}, \mathrm{H}^{13} \mathrm{CN}$, and $\mathrm{HC}^{15} \mathrm{~N}$. Our grid of models encompasses a range of $\mathrm{H}_{2}$ density $\left(10^{6}-10^{11} \mathrm{~cm}^{-3}\right)$ and kinetic temperature (from 5 to $30 \mathrm{~K}$ ) relevant to the TW Hya orbiting disk. The results of these calculations in terms of $T_{\text {ex }}$ are shown in Fig. C.1. There are significant departures at densities between $10^{6} \mathrm{~cm}^{-3}$ and $10^{8} \mathrm{~cm}^{-3}$. At higher density, all lines are thermalized (models at $n_{\mathrm{H}_{2}}$ higher than $10^{9} \mathrm{~cm}^{-3}$ are not shown). From the generic chemical models of Visser et al. (2018), it appears that $\mathrm{HCN}$ is concentrated at radii $r=5-50$ au and scale heights $z / r=0.2$ to 0.3 , where the gas kinetic temperature is $\approx 200 \mathrm{~K}$ and the density in the range $10^{6}$ to $10^{8} \mathrm{~cm}^{-3}$, which would indicate that the single excitation temperature assumption fails.

On the other hand, the FWHM of the $\mathrm{HCN}, \mathrm{H}^{13} \mathrm{CN}$, and $\mathrm{HC}^{15} \mathrm{~N}$ lines at a radius of $0.6^{\prime \prime}(\approx 35 \mathrm{au})$ and beyond is $\approx 0.35 \mathrm{~km} \mathrm{~s}^{-1}$ (see Table B.3), placing a conservative upper limit on the kinetic temperature of (Teague et al. 2016)

$(F W H M / 2.35)^{2} \times \mu m_{\mathrm{H}} /\left(2 k_{\mathrm{B}}\right) \approx 35 \mathrm{~K}$.

Referring to the tailored TW Hya model of van't Hoff et al. (2017), this upper limit, applied to the relevant range of radii, indicates that the density is higher than $10^{9} \mathrm{~cm}^{-3}$, in a regime where lines should be thermalized. This validates our single excitation temperature assumption. Our upper limit on the kinetic temperature is also well below the gas temperature of $\sim 200 \mathrm{~K}$ where the $\mathrm{HCN}$ abundance is the highest in the models of Visser et al. (2018), although we note that these models are generic and are not tailored to TW Hya. 